\title{
Agarose Gel Electrophoresis of Polyelectrolytes
}

\author{
Kensuke Shimizu, Naoki SaKaI, and Akira TaKaHaSHI \\ Department of Industrial Chemistry, Mie University, Tsu, Mie, 514, Japan
}

(Received February 7, 1990)

\begin{abstract}
Agarose gel electrophoresis of synthetic polyelectrolytes, sodium polystyrene sulphonate (NaPSS), poly(acrylic acid) sodium salts (Na-PA) and carboxymethyl cellulose sodium salts (CMC) was studied. Using NaPSSs which have narrow molecular weight distribution, depending on the relative dimensions of radii of gyration of polyions, $R_{\mathrm{g}}$, and pore size in the gel, $\bar{a}$, it was found that the electrophoretic migration mode is separated into three regimes, namely (1) Ogston $\left(R_{\mathrm{g}} \leq \bar{a}\right)$, (2) reptation without stretching $\left(R_{\mathrm{g}}>\bar{a}\right)$ and (3) reptation with stretching, $\left(R_{\mathrm{g}} \gg \bar{a}\right)$. These findings are quite similar to the agarose gel electrophoresis of DNA first oserved by Slater and Noolandi. Using Na-PSS as standard samples, it was found that, in the regime of reptation without stretching, the friction coefficient $f$ of a monomeric unit is proportional to the cube root of the molar volume of a monomer unit of Na-PA. For measurement of molecular weight of polyions with unknown molecular weight by gel electrophoresis, in the Ogston regime correction for the observed mobility is unnecessary if we use Na-PSS as a standard. However, in the regime (2), mobility should be corrected by evaluating the $f$ of unknown polyions.
\end{abstract}

KEY WORDS Agarose / Electrophoresis / Gel / Polyelectrolyte / Poly(acrylic acid) / Polystyrenesulphonate / Carboxy Methyl Cellulose / Reptation /

In order to separate as well as to characterize biological polyelectrolytes such as deoxyribonucleic acid (DNA) and sodium dodecyl sulfate denatured proteins, agarose gel electrophoresis was introduced in the 1960's. It was shown that the method is useful not only for determination of molecular weight but also for separation of DNA fragments depending on their sizes. However, it has often been observed that with increasing molecular size of DNA, electrophoretic separation of DNA depending on chain length becomes difficult. To overcome this shortcoming, pulse field gel electrophoresis has been introduced by Schwartz and Cantor. ${ }^{17}$ Then, intensive experimental and theoretical studies on gel electrophoresis of DNA began. However, the extension of this method to characterize synthetic polyelectrolytes has hardly been done. ${ }^{1-3}$

Ferguson $^{4}$ studied gel electrophoresis of pituitary proteins and polypeptides based on the investigation of Ogston in $1958 .{ }^{5}$ Ferguson derived an equation which correlates the electrophoretic mobility, $\langle\dot{x}\rangle / E$ in a gel in terms of the free mobility, $\mu_{0}$ in bulk solution, and agarose gel concentration, $C_{\mathrm{A}}{ }^{4,6}$ That is

$$
\log \langle\dot{x}\rangle / E=\log \mu_{0}-b C_{\mathrm{A}}\left(R_{\mathrm{g}}+r\right)^{2}
$$

where $b$ is a constant, $R_{\mathrm{g}}$ is the radius of gyration of polyions and $r$ is the radius of rod like fibers which construct the gel structure. ${ }^{5}$

However, this model is only effective for the migration of small polyions whose size is smaller than the average pore size $\bar{a}$ in the gel. Therefore, to explain electrophoretic behavior of larger size DNA, the reptation model was introduced by Zimm, Lumpkin, and Déjardi$\mathrm{n}^{7-9}$ and Slater and Noolandi. ${ }^{10-14}$

Furthermore, to generalize this model covering longer chain length of DNA, Slater, Noolandi, Rousseau, Turmel and Lalande ${ }^{15}$ differentiated the migration mode of DNA into three regimes by comparing the radii of gyration of DNA with the average pore size of 
agarose gel, $\bar{a}$, namely (1) Ogston regime ${ }^{4-6}$ where $R_{\mathrm{g}}<\bar{a}$, (2) reptation without stretching regime $^{7-14,16}\left(R_{\mathrm{g}}>\bar{a}\right)$ and (3) reptation with stretching regime ${ }^{7-14}\left(R_{\mathrm{g}} \gg \bar{a}\right)$.

It is considered that the theory is equally applicable to the gel electrophoresis of synthetic polyelectrolytes since the theory uses only the concept of segments.

The purpose of this work is (1) comparison of the agarose gel electrophoresis experiments of a synthetic polyelectrolytes, sodium polystyrene sulphonate with the theory ${ }^{1}$ and clarify the migration mechanism of the polyion, (2) establishment of the method to characterize polyelectrolytes whose molecular size is unknown.

\section{EXPERIMENTAL}

Five samples of sodium polystryene sulphonate (Na-PSS) with narrow molecular weight distributions were purchased from Pressure

Table I. Characteristics of sodium polystyrene sulphonates, sodium poly(acrylate)s, and sodium carboxy methyl celluloses

\begin{tabular}{|c|c|c|c|c|c|c|}
\hline & Code & \multicolumn{2}{|c|}{$M_{w} \times 10^{-3}$} & $M_{w} / M_{n}$ & \multicolumn{2}{|c|}{$D P$} \\
\hline \multirow[t]{8}{*}{ Na-PSS } & S1 & \multicolumn{2}{|c|}{88} & 1.05 & \multicolumn{2}{|c|}{430} \\
\hline & $\mathrm{S} 2$ & \multicolumn{2}{|c|}{177} & 1.05 & \multicolumn{2}{|c|}{860} \\
\hline & $\mathrm{S} 3$ & \multicolumn{2}{|c|}{354} & 1.05 & \multicolumn{2}{|c|}{1720} \\
\hline & S4 & \multicolumn{2}{|c|}{690} & 1.05 & \multicolumn{2}{|c|}{3350} \\
\hline & S5 & \multicolumn{2}{|c|}{1060} & 1.05 & \multicolumn{2}{|c|}{5150} \\
\hline & \multirow{2}{*}{$\begin{array}{l}\text { S6 } \\
\text { S7 }\end{array}$} & \multicolumn{2}{|c|}{2990} & - & \multicolumn{2}{|c|}{14510} \\
\hline & & \multicolumn{2}{|c|}{5360} & - & \multicolumn{2}{|c|}{26020} \\
\hline & S8 & \multicolumn{2}{|c|}{8050} & - & \multicolumn{2}{|c|}{39080} \\
\hline \multirow[t]{7}{*}{$\mathrm{Na}-\mathrm{PA}$} & Al & \multicolumn{2}{|c|}{53.7} & - & \multicolumn{2}{|c|}{570} \\
\hline & $\mathrm{A} 2$ & \multicolumn{2}{|c|}{141} & - & \multicolumn{2}{|c|}{1500} \\
\hline & A3 & \multicolumn{2}{|c|}{434} & - & & 600 \\
\hline & A4 & & 5 & - & & 200 \\
\hline & A5 & & 7 & - & & 350 \\
\hline & A6 & & 3 & - & & 200 \\
\hline & Code $M_{w}$ & $\times 10^{-3}$ & $M_{w}$ & $\begin{array}{r}\text { Degr } \\
\text { substi }\end{array}$ & $\begin{array}{l}\text { of } \\
\text { tion }\end{array}$ & $D P$ \\
\hline $\mathrm{Na}-\mathrm{CMC}$ & $\mathrm{Cl}$ & 326 & 1.4 & & & 1200 \\
\hline & $\mathrm{C} 2$ & 327 & 1.3 & & & 1150 \\
\hline & $\mathrm{C} 3$ & 393 & 1.7 & & & 1100 \\
\hline
\end{tabular}

Chemical Co. Three other Na-PSSs of higher molecular weight were fractionated samples and kindly donated from Prof. T. Kato and Mr. S. Usuki. Their characteristics are shown in Table I. Sodium salts of poly(acrylic acid) (Na-PA) (Toa Gosei Chem. Co.) and sodium carboxy methyl celluloses. (Na-CMC) (Daicel Chem. Co.) were fractionated samples and their characteristics are also shown in Table I.

Agarose (type HGT(p)), trishydroxy methyl amino methane and boric acid (all are electrophoresis grade) were purchased from Nacalai Tesque Co., and methylene blue was supplied from Nakarai Chem Co. Twice distilled water was used as the solvent.

\section{METHODS}

\section{Preparation of Agarose Gel}

Agarose was dissolved in $100 \mathrm{ml}$ of the tris-boric acid buffer solution $(\mathrm{pH}=8.1)$ by boiling. After cooling to approximately $60^{\circ} \mathrm{C}$, the agarose solution was poured into $15 \mathrm{~cm} \times$ $15 \mathrm{~cm}$ gel bed (Bio-Rad) equipped with a 15 well comb (Bio-Rad) with $0.55 \mathrm{~cm} \times 0.2 \mathrm{~cm}$ teeth. The gel was allowed to solidify for an hour at room temperature.

\section{Gel Electrophoresis}

Gel electrophoresis of polyions was performed by a Mitumi Model SJ-1051. The gel was placed in the submarine cell, and the cell was filled with tris-boric acid buffer solution. The $5.0 \times 10^{-2} \mathrm{ml}$ sample solution $(50 \mu \mathrm{g}$ of samples dissolved in the buffer solution) was injected into the well. Electrophoresis was carried out at various agarose gel concentrations under continuous electric fields $(E=1.2$ $\mathrm{V} \mathrm{cm}^{-1}$ ) during $2-40 \mathrm{~h}$.

After electrophoresis, the gel was immersed in the methylene blue $(0.25 \mathrm{wt} \%)$ solution for about $30 \mathrm{~min}$ and $\mathrm{Na}-\mathrm{PSS}$ as well as $\mathrm{Na}-\mathrm{PA}$ and $\mathrm{Na}-\mathrm{CMC}$ bands were visualized by staining. 


\section{RESULTS AND DISCUSSION}

The radii of gyration of Na-PSS in the tris-boric acid buffer solution were evaluated from the intrinsic viscosity of Na-PSS in the buffer solution by making use of the Flory-Fox equation $^{23}$

$$
[\eta]=\Phi \frac{R_{\mathrm{g}}{ }^{3}}{M}
$$

where $\Phi=1.5 \times 10^{21}$, since in general $\Phi$ for polyelectrolytes is somewhat lower than $2.1 \times 10^{21} \cdot{ }^{24}$ The results are shown in Table II.

After gel electrophoresis for the specified time $t(\mathrm{~h})$, positions of polyions were visualized by methylene blue staining, and then distances, $d(\mathrm{~cm})$ of the center of polyions bands from the comb position of the gel were measured. The average migration velocity of polyions $\langle\dot{x}\rangle$ is calculated as $\langle\dot{x}\rangle=d / t$ and the electrophoretic mobility is given by

$$
\langle\dot{x}\rangle / E=d /(t \cdot E) \quad\left(\mathrm{cm}^{2} \cdot \mathrm{v}^{-1} \cdot \mathrm{h}^{-1}\right)
$$

The experimental reproducibility of $\langle\dot{x}\rangle / E$ was $\pm 10 \%$.

Figure 1 shows logarithmic plots of mobility of Na-PSS $v s$. logarithm of $1 / P$, where $P$ is the degree of polymerization $(D P)$ of Na-PSS, for various agarose gel concentration. At $0.5 \%$ of agarose gel concentration, the mobilities increase linearly with decreasing $D P$ with the

Table II. Radii of gyration of sodium polystyrene sulphonates

\begin{tabular}{|c|c|c|c|}
\hline Code & $M_{w} \times 10^{-3}$ & $\begin{array}{c}{[\eta]} \\
\text { in tris-boric acid } \\
\text { buffer solution }\left(100 \mathrm{ml} \mathrm{g}^{-1}\right)\end{array}$ & $\frac{R_{\mathrm{g}}}{\AA}$ \\
\hline $\mathrm{S} 1$ & 88 & 0.20 & 120 \\
\hline $\mathrm{S} 2$ & 177 & 0.50 & 181 \\
\hline S3 & 354 & 0.88 & 277 \\
\hline S4 & 690 & 1.05 & 410 \\
\hline S5 & 1060 & 2.10 & 529 \\
\hline S6 & 2990 & 4.90 & 995 \\
\hline S7 & 5360 & 7.80 & 1413 \\
\hline S8 & 8050 & 10.7 & 1730 \\
\hline
\end{tabular}
estimated by eq 4 slope of 0.2 . However, above $1.0 \%$ of agarose gel concentration, the mobility first decreased with increasing $D P$ and then further decreased with the slope of 1 and finally reached a smaller slope line at very high $D P$.

Our experimental results for the elecrophoresis of Na-PSS are analyzed by the theory of Noolandi et al. ${ }^{15}$ in the next section.

\section{Comparison with the theory of Slater and Noolandi}

(1) Ogston Regime. If $R_{\mathrm{g}}$ in eq 1 is fixed, i.e., at a constant molecular weight of polyions, the plot of logarithm of mobility as a function of $C_{\mathrm{A}}$ is expected to give a straight line with a slope of $K_{\mathrm{r}}=b\left(R_{\mathrm{g}}+r\right)^{2}$ and an intercept of $\log \mu_{0}$, where $K_{\mathrm{r}}$ is known as the retardation coefficient. $^{15}$

In Figure 2, following eq 1 the logarithm of mobility is plotted against agarose gel concentration, which is generally known as the Ferguson plot. ${ }^{4}$ For samples of Na-PSS below $M_{w}=1.77 \times 10^{5}$, the plots are straight lines. However, further increase of molecular weight causes departure from linearity. Hence, for determination of $K_{\mathrm{r}}$, we took initial slopes of

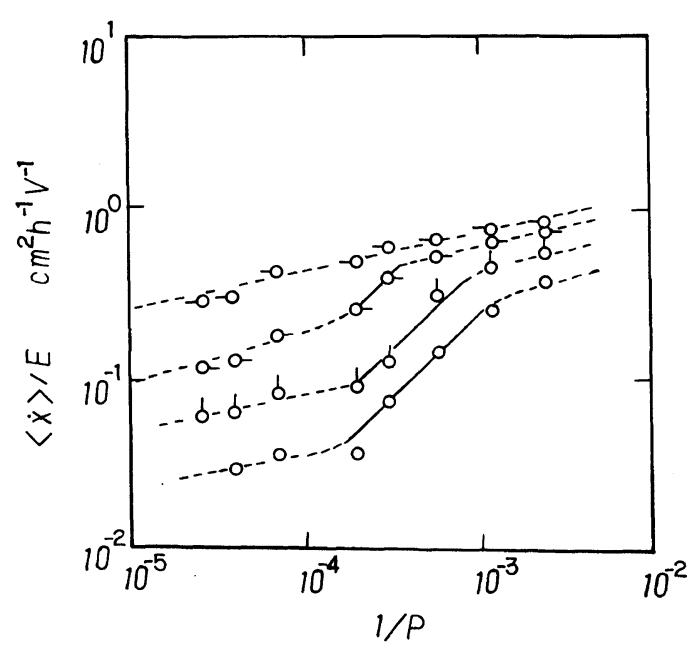

Figure 1. The logarithmic plot of mobility, $\langle\dot{x}\rangle / E$ of Na-PSS $v s$. logarithm of $1 / P$. Field strength, $E=1.2 \mathrm{~V}$ $\mathrm{cm}^{-1}$ and agarose gel concentration. $-\mathrm{O}, 0.5 \% ; \mathrm{O}-$ $1.0 \%, \bigcirc, 2.0 \% ; \bigcirc, 3.0 \%$. Solid lines have a slope of 1 . 


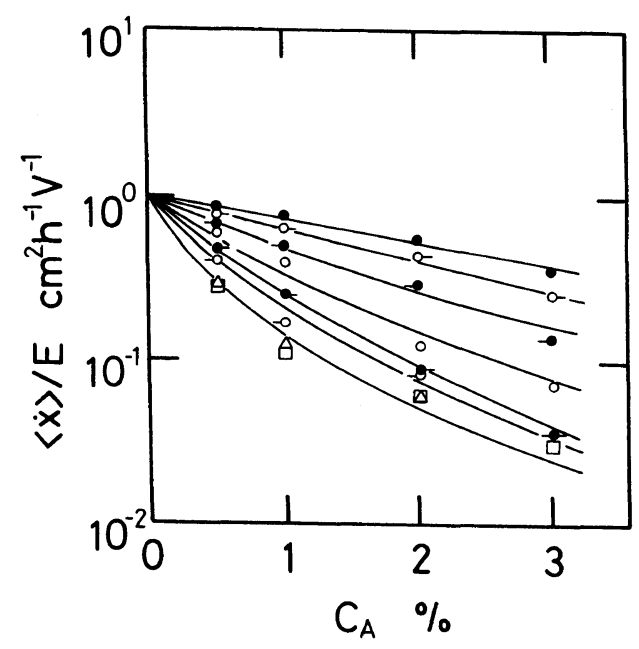

Figure 2. The logarithmic plots of mobility, $\langle\dot{x}\rangle / E$ of Na-PSS vs. agarose gel concentration. Field strength, $E=1.2 \mathrm{~V} \mathrm{~cm}^{-1}$ and molecular weight of Na-PSS, $M_{w} \times$ $10^{-3}: \bigcirc, 88.0 ; \bigcirc-, 177.0 ;-\bigcirc, 354.0 ; \bigcirc, 690.0 ; 0-$, $1060 ;-\bigcirc, 2990 ; \square, 5360 ; \triangle, 8050$.

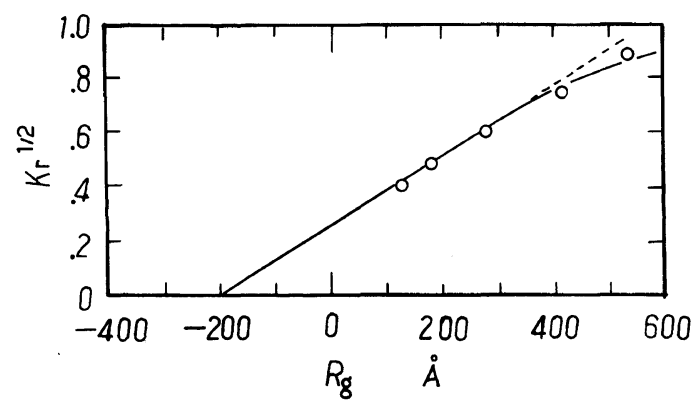

Figure 3. The relationship between the square root of retardation coefficient, $K_{\mathrm{r}}^{1 / 2}$ and radii of gyration, $R_{\mathrm{g}}$ of Na-PSS. The intercept of this plot at $K_{\mathrm{r}}{ }^{1 / 2}=0$ reveals the average fiber radii in agarose gel, $R=200 \AA$.

the curves in Figure 2.

Furthermore, the intercepts of the plots almost coincide at one point indicating that the electrophoretic mobility $\mu_{0}$ in the bulk solution is independent of molecular weight, which has already been confirmed for the electrophoresis of linear polyelectrolytes in bulk salt solution. ${ }^{18-22}$

Since $K_{\mathrm{r}}^{1 / 2}=b^{1 / 2}\left(r+R_{\mathrm{g}}\right)$ then the plot of $K_{\mathrm{r}}^{1 / 2}$ against $R_{\mathrm{g}}$ allows us to estimate the average fiber radius, and we obtained $r=200 \AA$

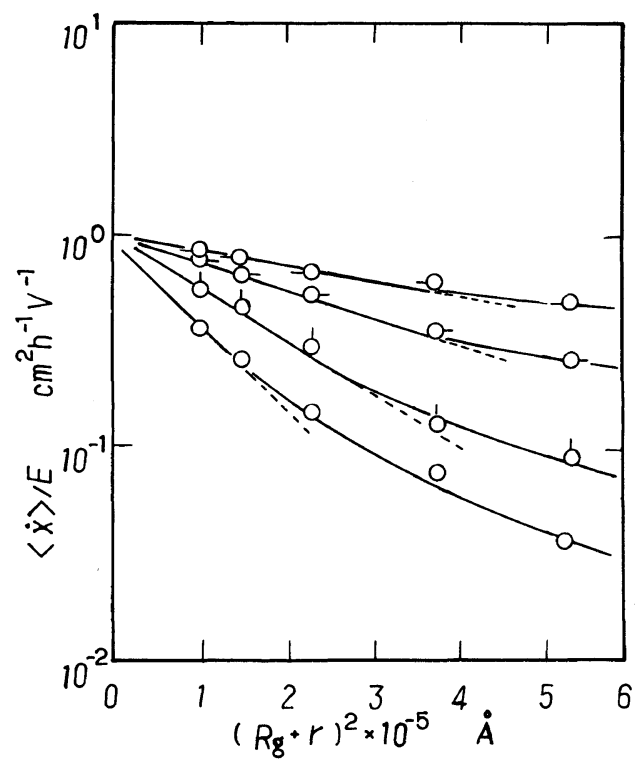

Figure 4. The logarithmic plots of mobility, $\langle\dot{x}\rangle / E$ of Na-PSS $v s$. $\left(R_{\mathrm{g}}+r\right)^{2}$. Field strength $E=1.2 \mathrm{~V} \mathrm{~cm}^{-1}$ and agarose gel concentration, $C_{\mathrm{A}}$ : $-\mathrm{O}, 0.5 \%$; $\bigcirc, 1.0 \%$; ○, $2.0 \% \bigcirc, 3.0 \%$.

Table III. Average pore size $\bar{a} \sim R_{\mathrm{g}}$ and gel concentration

\begin{tabular}{cc}
\hline$A / \%$ & $\bar{a} / \AA$ \\
\hline 0.5 & 500 \\
1.0 & 290 \\
2.0 & 150 \\
3.0 & 80 \\
\hline
\end{tabular}

in the agarose gel at $K_{\mathrm{r}}^{1 / 2}=0$ as shown in Figure 3.

Upon evaluated $\mathrm{r}$ and $R_{\mathrm{g}}$, in Figure 4 , the mobility is plotted against $\left(R_{\mathrm{g}}+r\right)^{2}$ (eq 1$)$ and the plots are linear for smaller size of Na-PSS, while, for larger size of NaPSS, the plots deviate from straight line.

The average pore diameter $\bar{a}$ may be evaluated by the method of Ogston ${ }^{4,15}$ if one knows $R_{\mathrm{g}}$ of polyions. According to the theory, at $R_{\mathrm{g}} \simeq \bar{a}$, the following relationship holds

$$
(\langle\dot{x}\rangle / E) / \mu_{0} \simeq 1 / 2
$$

The results of estimation of $\bar{a}$ are tabulated 
in Table III. Thus, it may be concluded that the mobility of sufficiently small size of polyion belongs to Ogston regime.

(2) Reptation Regime. The mobility of polyions in reptation regime ${ }^{7-14}$ is given by

$$
\langle\dot{x}\rangle / E \cong(Q / 3 \zeta)\left[(1 / P)+\left(E^{\prime 2} / 3\right)\right]
$$

where $Q$ is the total charge on a polyion, $\zeta$ is the friction coefficient for translational motion of the whole chain along the tube, and $E^{\prime}$ is the dimensionless reduced electric field defined by $E^{\prime}=q E l / 2 k T$ whre $q$ is the effective charge on a polyion segment and $l$ is the length of a segment of the tube. Since $\zeta=f P$, where $f$ is the friction coefficient of a monomer unit and $Q \propto P$, the term $Q / 3 \zeta$ is independent of molecular weight of polyions.

According to eq 5, if the electric field strength is sufficiently weak and the first term is much larger than the second term in the square bracket, eq 5 is approximated by

$$
\langle\dot{x}\rangle / E \cong(Q / 3 \zeta) \cdot(1 / P)
$$

Therefore, in Figure 1, the regions where the double logarithmic plot of $\langle\dot{x}\rangle \mid E v s .1 / P$ has the slope of 1 belongs to the reptation regime without stretching. For example to $3 \%$ gel, the range of molecular weight is from $1.77 \times 10^{5}$ to $1 \times 10^{6}$.

(3) Reptation Regime with Stretching. When the polyion size becomes extremely large, then $1 / P$ is negligibly small compared with $E^{\prime}$ and the polyion size-dependence of the mobility will disappear. ${ }^{7-14}$

When $M_{w}>10^{6}$ or $D P>10^{4}$, then experimental observation shown in Figure 1 almost coincides with this expectation. However we do not have any direct evidence of the stretching of polyions at the present state. Judging from the comparison between average pore size $\bar{a}$ and radius of gyration of polyions of extremely high molecular weight of Na-PSS, the polyion will make a column of connection of blobs in the tube which restricts the polyion. The end-to-end distance of a polyion in the tube is much larger than that of unrestricted

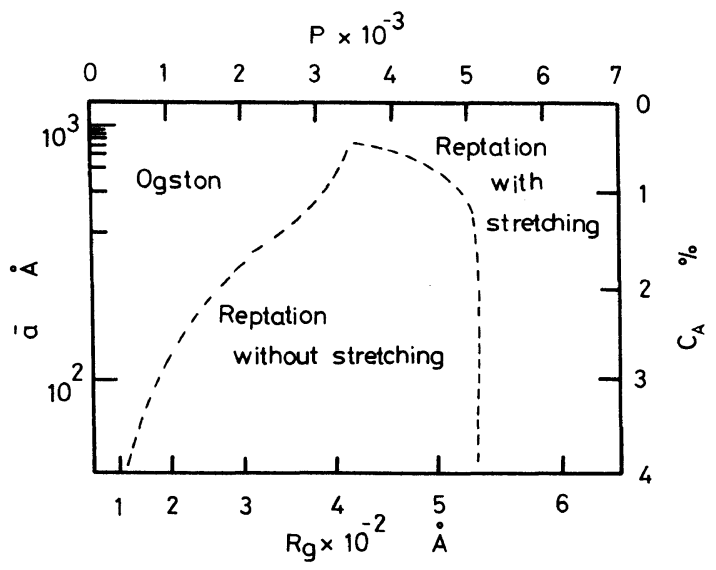

Figure 5. The phase diagram of the agarose gel electrophoresis of Na-PSS at field strength, $E=1.2 \mathrm{~V} \mathrm{~cm}^{-1}$. Degree of polymerization, $P$ and the radii of gyration, $R_{\mathrm{g}}$ (in $\AA$ ) are given on the upper and lower $x$ axes. Agarose gel concentration, $C_{\mathrm{A}}$ (in \%) and the corresponding average pore size $\bar{a}$ (in $\AA$ ) are given on the left and right $y$ axes.

polyion i.e., a random coil conformation.

\section{Phase Diagram}

The Phase diagram of DNA gel electrophoresis, which indicates three regimes, has been given by Slater and Noolandi et al. Following them, the phase diagram for gel electrophoresis of Na-PSS is constructed as Figure 5, which also indicates three regimes, namely Ogston, reptation and stretched reptation.

The Ogston-to-reptation transion point was determined from the onset of nonlinearity on Ferguson plot (Figure 2) and the reptation-tostretched reptation transition point was determined from the extrapolated intersection of both reptation and streched reptation region (Figure 1).

We can easily find correlations among polyion sizes (molecular weights as well as radii of gyration) and gel concentration with pore size information. The diagram is very useful for molecular characterization of polyions of unknown molecular weight and molecular weight distribution. 
III. Calibration Method to Characterize Polyions with unknown Molecular Weight

In the characterization of polyion sizes, the merit of gel electrophoresis experiments is its easiness for comparison with measurements of intrinsic viscosity and/or gel permeation chromatography. However, the gel electrophoresis does not offer absolute mobility which depends on molecular size of polyions. Hence, we must have standard samples of known molecular weight in performing molecular characterization of polyelectrolytes of unknown molecular size as well as molecular weight distribution. ${ }^{2,3}$ As standard samples, Na-PSS samples are quite appropriate since they have narrow molecular weight distribution. Using Na-PSS as a standard, the electrophoretic mobilities were measured for $\mathrm{Na}-\mathrm{PA}$ as well as $\mathrm{Na}-\mathrm{CMC}{ }^{1}$

In Table IV, the apparent degree of polymerization $P_{\text {app }}$ is compared with the

Table IV.

(a) Ogston regime

(1) Na-PA

\begin{tabular}{rc}
\hline$D P^{\mathrm{a}}$ & $P_{\mathrm{app}}=D P_{\mathrm{c}}$ \\
\hline 570 & 600 \\
1500 & 1200 \\
\hline
\end{tabular}

(2) $\mathrm{Na}-\mathrm{CMC}$

\begin{tabular}{cc}
$D P$ & $P_{\text {app }}$ \\
\hline 1200 & 1100 \\
1150 & 1250 \\
1100 & 1400 \\
\hline
\end{tabular}

(b) Reptation regime

\begin{tabular}{ccc}
\multicolumn{3}{c}{ Na-PA } \\
\hline$D P$ & $P_{\text {app }}$ & $\begin{array}{c}\text { Corrected } D P_{\mathrm{c}}= \\
P_{\text {app }} / f_{\text {sp }}\end{array}$ \\
\hline 4600 & 3200 & 4600 \\
5200 & 3000 & 4200 \\
6350 & 3800 & 5400 \\
8200 & 5000 & 7250 \\
\hline
\end{tabular}

a $D P$ from Table I. degree of polymerization $D P$. It is clear that for Na-CMC and low molecular weight $\mathrm{Na}-\mathrm{PA}$, which belongs to the Ogston regime, the agreement between $P_{\text {app }}$ and $D P$ is good, and therefore we can use Na-PSS as a molecular weight standard without applying any correction.

In the reptation regime, however, $P_{\text {app }}$ of Na-PA is smaller than the true DP of Na-PA. This indicates that the careless use of Na-PSS as a standard for molecular weight determination of uncharacterized polyions with different molecular structure leads to erroneous results. In this regime, it should be noted that $\langle\dot{x}\rangle \mid E$ is a function not only of $1 / P$ but also $1 / f,^{7-14}$ i.e.,

$$
\langle\dot{x}\rangle \mid E \propto \frac{1}{f P}
$$

Therefore, we must correct the difference of the friction coefficient of a monomer unit of Na-PSS and Na-PA since the sizes of monomeric unit of both polyions are different. Assuming that the friction coefficient is proportional to the radius of a monomer unit, $f$ is proportional to cube root of molar volume of a monomer unit, i.e.,

$$
f \propto r \propto V^{1 / 3}
$$

and taking the ratio of $f$ for both polyions, we define the specific friction coefficient $f_{\mathrm{sp}}$ as

$$
f_{\mathrm{sp}}=r_{\mathrm{PA}} / r_{\mathrm{PSS}}=\left(V_{\mathrm{PA}} / V_{\mathrm{PSS}}\right)^{1 / 3}
$$

where $V_{\mathrm{PA}}$ and $V_{\mathrm{PSS}}$ are the molar volumes of a monomer unit of Na-PA and Na-PSS, respectively. Amoore's method ${ }^{25}$ was employed to evaluate $V$ and we obtained

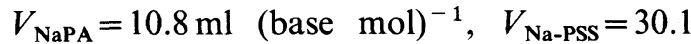
$\mathrm{ml}$ (base mol) ${ }^{-1}$, respectively and $f_{\mathrm{sp}}=0.7$. Multiplying $f_{\mathrm{sp}}$ to the observed mobility of Na-PA will give corrected $D P_{\mathrm{c}}$ of Na-PA and the results are tabulated in the third column of Table IV. The agreement between the corrected $D P_{\mathrm{c}}$ and $D P$ is fair. 


\section{CONCLUSIONS}

The agarose gel electrophoresis of synthetic polyelectrolyte revealed that the migration mechanism is separated into three regimes, namely for a 3\% gel (1) Ogston regime $\left(D P<10^{3}\right)$, (2) reptation without stretching regime $\left(10^{3}<D P<10^{4}\right)$ and (3) reptation with stretching regime $\left(D P>10^{4}\right)$, which is in conformity with the theory of Slater and Noolandi for DNA electrophoresis.

The phase diagram which offers a very useful guide when one wants to perform the agarose gel electrophoresis of polyions is constructed.

For molecular size determination of polyions of unknown characteristics, Na-PSS having narrow molecular weight distribution is a very good standard. If the sizes of the polyions are in the Ogston regime, no correction is necessary to obtain molecular size. However, if the migration velocity is in the reptation regime, correction for the frictional coefficient of a monomeric unit is necessary.

\section{REFERENCES}

1. A. Takahashi and N. Sakai, Polym. Prepr., Jpn., 36, 1037 (1987).

2. J. L. Chen and H. Morawetz, Macromolecules, 15, 1185 (1982).

3. D. L. Smisek and D. A. Hoagland, Macromolecules, 22, 2270 (1989).

4. K. A. Ferguson, Metabolism, 13, 985 (1964).

5. A. G. Ogston, Trans. Faraday. Soc., 54, 1754 (1958).
6. D. Rodbard and A. Chrambach, Proc. Natl. Acad. Sci., 65, 970 (1970).

7. O. J. Lumpkin and B. H. Zimm, Biopolymers, 21, 995 (1982).

8. O. J. Lumpkin, P. Dejardin, and B. H. Zimm, Biopolymers, 24, 1573 (1985).

9. O. J. Lumpkin, and B. H. Zimm, Biopolymers, 21, 2315 (1982).

10. G. W. Slater and J. Noolandi, Biopolymers, 24, 2181 (1985).

11. G. W. Slater and J. Noolandi, Phys. Rev. Lett., 55, 1579 (1985).

12. G. W. Slater and J. Noolandi, Biopolymers, 25, 431 (1986).

13. G. W. Slater, J. Rousseau, and J. Noolandi, Biopolymers, 26, 863 (1987).

14. G. W. Slater and J. Noolandi, Polym. Prepr., 29, 416 (1988).

15. G. W. Slater, J. Noolandi, J. Rousseau, C. Turmel, and M. Lalande, Biopolymers, 27, 509 (1988).

16. M. Doi and S. F. Edwards, J. Chem. Soc., Faraday Trans. II, 74, 1789 (1978).

17. D. C. Schwartz and C. R. Cantor, Cell, 37, 67 (1984).

18. M. Nagasawa, A. Soda, and I. Kagawa, J. Polym. Sci., 31, 439 (1958).

19. I. Noda, M. Nagasawa, and M. Ota, J. Am. Chem. Soc., 86, 5075 (1964).

20. T. Takahashi, I. Noda, and M. Nagasawa, J. Phys. Chem., 74, 1280 (1970).

21. M. Nagasawa, I. Noda, T. Takahashi, and N. Shimamoto, J. Phys. Chem., 76, 2286 (1972).

22. K. Terajima, T. Takahashi, and M. Nagasawa, Polym. J., 8, 449 (1976).

23. P. J. Flory, "Principles of Polymer Chemistry," Cornell Univesity Press, Ithaca, N.Y., 1953, Chapter 14.

24. A. Takahashi, T. Kato and M. Nagasawa, J. Phys. Chem., 71, 2001 (1967).

25. J. E. Amoore, Ann. N.Y. Acad. Sci., 116, 457 (1964). 